\title{
Uncontrolled and apparent treatment resistant hypertension: a cross-sectional study of Russian and Norwegian 40-69 year olds
}

Jakob Petersen ${ }^{1 *}$, Sofia Malyutina ${ }^{2,3}$, Andrey Ryabikov $^{3}$, Anna Kontsevaya ${ }^{4}$, Alexander V. Kudryavtsev ${ }^{5}$, Anne Elise Eggen ${ }^{6}$, Martin McKee ${ }^{1}$, Sarah Cook ${ }^{6}$, Laila A. Hopstock ${ }^{6}$, Henrik Schirmer ${ }^{6,7,8}$ and David A. Leon ${ }^{1,6,9}$

\begin{abstract}
Background: Uncontrolled hypertension is a major cardiovascular risk factor. We examined uncontrolled hypertension and differences in treatment regimens between a high-risk country, Russia, and low-risk Norway to gain better understanding of the underlying factors.

Methods: Population-based survey data on 40-69 year olds with hypertension defined as taking antihypertensives and/or having high blood pressure $(140+/ 90+\mathrm{mmHg})$ were obtained from Know Your Heart Study $(\mathrm{KYH}, \mathrm{N}=2284)$, Russian Federation (2015-2018) and seventh wave of The Tromsø Study (Tromsø 7, N = 5939), Norway (2015-2016). Uncontrolled hypertension was studied in the subset taking antihypertensives ( $\mathrm{KYH}: N=1584$; Troms $\varnothing$ 7: 2792)and defined as having high blood pressure $(140+/ 90+\mathrm{mmHg})$. Apparent treatment resistant hypertension (aTRH) was defined as individuals with uncontrolled hypertension on 3+ OR controlled on 4+ antihypertensive classes in the same subset.
\end{abstract}

Results: Among all those with hypertension regardless of treatment status, control of blood pressure was achieved in 22\% of men ( $\mathrm{KYH}$ and Tromsø 7), while among women it was 33\% in Tromsø 7 and $43 \%$ in $\mathrm{KYH}$. When the analysis was limited to those on treatment for hypertension, the percentage uncontrolled was higher in $\mathrm{KYH}(47.8 \%$, Cl 95 44.6-50.9\%) than Tromsø 7 (38.2, 36.1-40.5\%). The corresponding figures for aTRH were 9.8\% (8.2-11.7\%) and $5.7 \%(4.8-6.8 \%)$.

Antihypertensive monotherapies were more common than combinations and used by 58\% in Troms $\varnothing 7$ and $44 \%$ in $\mathrm{KYH}$. In both KYH and Tromsø 7, untreated hypertension was higher in men, those with no GP visit in the past year and problem drinkers. In both studies, aTRH was associated with older age, CVD history, obesity, and diabetes. In Tromsø 7, also male gender and any drinking. In KYH, also chronic kidney disease.

Conclusion: There is considerable scope for promoting combination therapies in line with European treatment guidelines in both study populations. The factors associated with untreated hypertension overlap with known correlates of treatment non-adherence and health check non-attendance. In contrast, aTRH was characterised by obesity and underlying comorbidities potentially complicating treatment.

Keywords: Cardiovascular diseases, Hypertension, Antihypertensive agents

\footnotetext{
* Correspondence: j.petersen@ucl.ac.uk

'London School of Hygiene \& Tropical Medicine, WC1E 7HT, London, UK

Full list of author information is available at the end of the article
} 


\section{Background}

Mortality from cardiovascular disease (CVD) has been falling rapidly in Russia since 2005 [1]. This is thought to be due, in part, to better detection, treatment, and control of hypertension [2-4]. Yet, despite this impressive progress, control of blood pressure remains relatively poor and there is a persisting mortality gap with countries of Western Europe. At first sight, there is no good reason for this. Russia has an extensive health system with, in comparative terms, large numbers of health workers [5]. Hypertension is easily diagnosed and can be treated with a range of safe and effective medicines [6], widely available in Russian pharmacies [7].

An effective response is clearly needed but this must be informed by a detailed understanding of why Russia has been unable to achieve better control of blood pressure. This study seeks to inform such a response by examining in detail the characteristics of a population sample of Russians, who have been initiated on antihypertensive treatment but whose blood pressure remains uncontrolled. It compares them with similarly defined individuals from neighbouring Norway to assess whether there are lessons that can be learnt from its comparatively better control of blood pressure.

In the study we compare uncontrolled and apparent treatment resistant hypertension among those taking antihypertensives in population-based samples from Russia and Norway as well as the proportion of individuals achieving blood pressure control among all with hypertension.

\section{Methods}

\section{Data selection criteria}

Know Your Heart (KYH) is a cross-sectional, populationbased study of cardiovascular structure, function, and risk factors in over 4500 men and women aged 35-69 years living in two Russian cities, Arkhangelsk and Novosibirsk, 2015-2018 [8]. The Tromsø Study is a longitudinal, population-based, prospective study with repeated data collections since 1974 in the municipality of Troms $\varnothing$ in Northern Norway. Data from the seventh wave of the study, Tromsø 7 (2015-2016), were used [9].

A total of 2284 and 5939 participants were selected for this study from $\mathrm{KYH}$ and Tromsø 7, respectively, based on the following inclusion criteria: aged 40-69 years, non-missing systolic and diastolic blood pressure measurement, measured hypertension (systolic pressure of $140+\mathrm{mmHg}$ and/or a diastolic of $90+\mathrm{mmHg}$ ) or taking antihypertensives [6]. A subset of participants, those taking antihypertensives (1584 from $\mathrm{KYH}$ and 2792 from Tromsø 7), were then selected to study uncontrolled and apparent treatment resistant hypertension. A flow diagram of the sample selection is presented in Figure S1 (Supplementary materials). Response rates for the health check component in $\mathrm{KYH}$ was $67 \%$ in Arkhangelsk and
37\% in Novosibirsk [8] (denominator: all issued, excluding addresses not found or where no one of target age and gender were found). The response rate in Tromsø 7 was $65 \%$ [9].

\section{Blood pressure measurement}

In $\mathrm{KYH}$, blood pressure was measured using OMRON 705 IT blood pressure monitors (OMRON Healthcare, Kyoto, Japan). All devices were calibrated before and after the fieldwork period and no adjustments were needed. Blood pressure in the Tromsø 7 study was measured using Dinamap ProCare 300 blood pressure monitors (GE Healthcare, Oslo, Norway) calibrated before the fieldwork. In both studies three measurements were taken with two minutes seated rest in between [8]. Participants were assigned to different antihypertensive classes based on their systolic and diastolic blood pressure (average of last two out of three measurements) according to the European hypertension treatment guidelines [6] and antihypertensive use.

\section{Medication}

Participants in both Know Your Heart (KYH, Russian Federation) and Tromsø 7 (Norway) were asked questions about their use of antihypertensives, although the protocols differed in some minor respects. In $\mathrm{KYH}$, a baseline interview was administered by a trained nonmedical interviewer. Participants who reported ever being diagnosed with hypertension were asked a series of questions about prescription and use of blood pressure medication. At the end of the interview all participants were invited to attend a health check to which they were asked to bring all their medications. At the health check, a trained medical interviewer asked the participant about current medication use and recorded the name, dose, indication and frequency of use of medications (up to 7 medications). About a third (33\%) of $\mathrm{KYH}$ participants brought their medicines with them to the health assessment and for the remaining two thirds (67\%), the record taking was verbatim. In Tromsø 7, all participants were asked about current or previous use of antihypertensive medications. Furthermore, participants were asked to state the name of all medications (prescription and nonprescription drugs) they had used regularly during the last four weeks (up to 20 medications). The questionnaire was checked by a trained technician at the study site, and participants had to confirm if no medication use was reported. For both studies listed medications were coded using the International WHO Anatomical Therapeutic Chemical (ATC) classification system [10]. Antihypertensive medication was defined as medications within the following ATC classes: Antihypertensives (abbreviation: AH; ATC code: C02), Diuretics (DIU; C03), Beta blockers (BB; C07), Calcium channel blockers 
(CCB; C08), ACE inhibitors (ACE; C09A/B), Angiotensin II receptor blockers (ARB; C09C/D).

The main analyses were based on ATC coding of reported medications. Sensitivity analyses were conducted using self-reported positive response to an explicit question about whether the participant regularly took antihypertensive medication.

\section{Outcome}

Uncontrolled hypertension was studied in those on antihypertensive medication in two different ways. First, as uncontrolled hypertension in the usual sense, i.e. individuals on antihypertensives and with systolic blood pressure $140+\mathrm{mmHg}$ and/or a diastolic of $90+\mathrm{mmHg}$. Second, as apparent treatment resistant hypertension (aTRH), i.e. individuals with uncontrolled hypertension on 3+ OR controlled on 4+ antihypertensive classes [11]. In a clinical setting, a patient would only be diagnosed with treatment resistant hypertension following a medication review, an assessment of medication adherence, and a series of elevated out-of-office blood pressure measurements. All of these factors are rarely present in large epidemiological studies and aTRH has shown to provide useful insights in various studies including NHANES studies $[11,12]$.

The prevalence of uncontrolled hypertension and aTRH among those with treated hypertension was estimated and standardised by age and sex to the 2013 European Standard Population [13].

\section{Co-variates}

A range of co-variates was included to study factors associated with hypertension, CVD risk, CVD prevention, and healthcare system use, i.e. gender, age, education, whether living with a partner, body mass index, alcohol use, CVD history, and primary healthcare visits. The equivalent covariates from $\mathrm{KYH}$ and Troms $\varnothing 7$ were harmonised in terms of coding frames and standard classifications.

Chronic kidney disease (CKD) status was defined as Glomerular Filtration Rate (eGFR) below $60 \mathrm{ml} / \mathrm{min} /$ $1.73 \mathrm{~m}^{2}$ based on serum creatinine [14].

Self-reported alcohol-related behaviours were categorised as: non-drinker past year versus low risk drinkers (score $<8$ ) versus high risk drinkers (score $8+$ ) according to WHO Alcohol Use Disorders Identification Test (AUDIT) [15].

For $\mathrm{KYH}$, the responses to a question on household financial constraints were classed into the following categories: perceived to be constrained in buying food or clothes, able to buy food or clothes but constrained in buying large domestic appliances, able to buy both. No equivalent variable was available from Tromsø 7.

The presence of diabetes was ascertained on either self-reported diabetes or taking diabetes medication
(ATC A10: insulin or oral antidiabetics) or HbA1c $48+$ $\mathrm{mmol} / \mathrm{mol}(>6.5 \%)$ [16].

Serum total cholesterol $(\mathrm{mmol} / \mathrm{L})$ was combined with data on age, sex, smoking status, and systolic blood pressure to calculate 10-year risk of a fatal CVD event according to the SCORE tool equations for high risk countries and divided into three risk groups for the descriptive analyses: Low (<1\%), Moderate (1-4.9\%), High $(5+\%)$ [17] (pers.comm. Dr. T. Fitzgerald for additional information regarding the Conroy et al. (2003) risk equations in line with the European Cardiology Society's online CVD risk calculator).

A history of CVD was defined as self-report of one or more of the following conditions: myocardial infarction, heart failure, atrial fibrillation, angina, stroke.

Whether the participant had visited a primary care consultant in the past year was also included (KYH: general practice or polyclinic; Tromsø 7: general practice).

Biomarker data from KYH (total cholesterol, HbA1c, serum creatinine) were corrected for inter-laboratory variation (Iakunchykova O, Averina M, Wilsgaard T, Leon DA: Recalibration of Blood Analytes in Know Your Heart Study for Comparisons with Tromsø 7 study - Impact of Recalibration on Mean Levels and Prevalence estimates, in preparation).

\section{Regression analyses}

Multivariate logistic regression models of uncontrolled versus controlled hypertension and aTRH versus nonaTRH in 40-69 year olds with treated hypertension were fitted adjusting for gender and age, or gender, age, and CVD history while including the following covariates: study, education, body mass index, smoking, alcohol consumption, diabetes, CKD, and primary healthcare visits. Statistical analyses were carried out using Stata 15 [18]. CVD history was included in the adjusted analyses together with gender and age due to the well-known differences in CVD burden between the two countries and the higher propensity for being and staying on antihypertensives for this group [19]. Potential interactions between age group, gender, age group, and CVD history for the two outcomes were tested with likelihood ratio tests in a restricted versus unrestricted model scenario.

\section{Results}

A total of 1584 and $279240-69$ year olds were on antihypertensives in $\mathrm{KYH}$ and Tromsø 7, respectively (for the characteristics of study participants, see Table 1). Individuals in Russia were more likely to have uncontrolled hypertension, at 55.7\% (95\% CI 50.6-60.6) in males and $42.7 \%$ (38.9-46.7) in females than in Norway (Table 2), where the corresponding percentages were $43.6 \%$ (40.4$46.8)$ and $33.0 \%$ (30.2-35.9). The proportion of people with hypertension who had aTRH was also higher in 
Table 1 Characteristics of participants by study (age- and sex-standardised to 2013 European Standard Population)

\begin{tabular}{|c|c|c|c|c|c|}
\hline \multirow[t]{2}{*}{ Characteristic } & \multirow[t]{2}{*}{ Level } & \multicolumn{2}{|l|}{$\mathrm{KYH}$} & \multicolumn{2}{|c|}{ Tromsø 7} \\
\hline & & $\mathrm{N}$ & $\%$ & $\mathrm{~N}$ & $\%$ \\
\hline Study total & Total & 1584 & 100 & 2792 & 100 \\
\hline \multirow[t]{6}{*}{ Age group } & $40-44 \mathrm{yr}$ & 80 & 5.1 & 151 & 5.4 \\
\hline & $45-49 y r$ & 125 & 7.9 & 273 & 9.8 \\
\hline & $50-54 \mathrm{yr}$ & 198 & 12.5 & 334 & 12.0 \\
\hline & $55-59 y r$ & 296 & 18.7 & 502 & 18.0 \\
\hline & $60-64 \mathrm{yr}$ & 405 & 25.6 & 720 & 25.8 \\
\hline & $65-69 y r$ & 480 & 30.3 & 812 & 29.1 \\
\hline \multirow[t]{2}{*}{ Gender } & Male & 598 & 38.0 & 1454 & 50.8 \\
\hline & Female & 986 & 62.0 & 1338 & 49.2 \\
\hline \multirow[t]{4}{*}{ Education } & Elementary & 245 & 12.9 & 744 & 21.7 \\
\hline & Lower intermediate & 204 & 12.1 & 891 & 33.5 \\
\hline & Higher intermediate & 652 & 42.4 & 524 & 20.5 \\
\hline & Graduate & 483 & 32.5 & 611 & 24.4 \\
\hline \multirow[t]{5}{*}{ Economic activity } & Paid work & 248 & 34.5 & 1668 & 71.5 \\
\hline & Looking after home & 152 & 7.1 & 16 & 0.4 \\
\hline & Unemployed & 24 & 2.8 & 15 & 0.7 \\
\hline & Retired & 1145 & 53.9 & 551 & 10.0 \\
\hline & Other & 15 & 1.6 & 504 & 17.3 \\
\hline \multirow[t]{3}{*}{ Financial constraints } & Constrained & 365 & 22.0 & N/A & N/A \\
\hline & Intermediary & 800 & 50.2 & N/A & N/A \\
\hline & Rel. unconstrained & 398 & 27.8 & N/A & N/A \\
\hline \multirow[t]{2}{*}{ Single } & No & 1039 & 67.4 & 2064 & 77.8 \\
\hline & Yes & 545 & 32.6 & 595 & 22.2 \\
\hline \multirow[t]{2}{*}{ Smoking } & No & 1262 & 74.4 & 2394 & 87.0 \\
\hline & Yes & 320 & 25.6 & 356 & 13.0 \\
\hline \multirow[t]{3}{*}{ Alcohol use disorder } & Non-drinker past year & 1145 & 67.6 & 1398 & 52.3 \\
\hline & Low (AUDIT< <) & 284 & 20.8 & 865 & 33.9 \\
\hline & High (AUDIT 8+) & 150 & 11.5 & 322 & 13.8 \\
\hline \multirow[t]{4}{*}{ Body Mass Index } & Under/Normal $(<25)$ & 226 & 15.7 & 498 & 17.3 \\
\hline & Overweight (25-29) & 572 & 34.3 & 1167 & 39.0 \\
\hline & Obese (30-34) & 489 & 29.8 & 765 & 28.5 \\
\hline & Very obese (35+) & 293 & 20.1 & 344 & 15.2 \\
\hline \multirow[t]{2}{*}{ Diabetic } & No & 1182 & 79.7 & 2366 & 84.9 \\
\hline & Yes & 402 & 20.3 & 426 & 15.1 \\
\hline \multirow[t]{3}{*}{ SCORE 10-year CVD risk\% } & Low $(<1 \%)$ & 232 & 33.8 & 466 & 34.7 \\
\hline & Intermediate (1-4.9\%) & 755 & 44.5 & 1325 & 45.6 \\
\hline & High (5+\%) & 564 & 21.7 & 949 & 19.7 \\
\hline \multirow[t]{2}{*}{ Seen GP past year } & No & 301 & 19.1 & 192 & 7.1 \\
\hline & Yes & 1283 & 80.9 & 2589 & 92.9 \\
\hline \multirow[t]{2}{*}{ CKD } & No & 1516 & 96.7 & 2694 & 97.2 \\
\hline & Yes & 68 & 3.3 & 98 & 2.8 \\
\hline \multirow[t]{2}{*}{ CVD history } & No & 910 & 65.9 & 2100 & 79.5 \\
\hline & Yes & 674 & 34.1 & 692 & 20.5 \\
\hline
\end{tabular}


Table 2 Prevalence of uncontrolled and an apparent treatment resistant hypertension (aTRH) and odds ratios (AOR; 95\% Cl) for inter-study effects

\begin{tabular}{|c|c|c|c|c|c|c|}
\hline \multirow[t]{2}{*}{ Class } & & \multirow[t]{2}{*}{ KYH (Russia) } & \multirow[t]{2}{*}{$\begin{array}{l}\text { Tromsø } 7 \\
\text { (Norway) }\end{array}$} & \multirow{2}{*}{$\begin{array}{l}\text { KYH:Troms } \varnothing \\
7 \text { AOR } \\
\text { Age/Sex }\end{array}$} & \multirow{2}{*}{$\begin{array}{l}\text { KYH:Tromsø } \\
7 \text { AOR } \\
\text { Age/Sex/CVD }\end{array}$} & \multirow{2}{*}{$\begin{array}{l}\text { KYH:Troms } \varnothing \\
7 \text { AOR } \\
\text { Multiple }^{\text {b }}\end{array}$} \\
\hline & & & & & & \\
\hline \multirow[t]{4}{*}{ Uncontrolled hypertension } & Male & 55.7 (50.6 to 60.6) & 43.6 (40.4 to 46.8$)$ & $1.90(1.56 ; 2.30)$ & $2.03(1.67 ; 2.47)$ & $1.97(1.59 ; 2.44)$ \\
\hline & Female & 42.7 (38.9 to 46.7 ) & 33.0 (30.2 to 35.9$)$ & $1.30(1.10 ; 1.54)$ & $1.35(1.14 ; 1.61)$ & $1.22(1.01 ; 1.49)$ \\
\hline & Total & 47.8 (44.6 to 50.9 ) & 38.2 (36.1 to 40.5$)$ & $1.54(1.36 ; 1.75)$ & $1.65(1.45 ; 1.87)$ & $1.59(1.39 ; 1.83)$ \\
\hline & Total $^{a}$ & 48.7 ( 45.2 to 52.2$)$ & 41.7 (39.5 to 44.0$)$ & N/A & N/A & N/A \\
\hline \multirow[t]{4}{*}{ aTRH } & Male & $10.8(8.1$ to 14.1$)$ & 6.9 (5.4 to 8.7$)$ & $1.62(1.18 ; 2.21)$ & $1.50(1.09 ; 2.06)$ & $1.58(1.12 ; 2.24)$ \\
\hline & Female & $9.2(7.3$ to 11.6$)$ & 4.5 (3.4 to 5.9$)$ & $2.05(1.50 ; 2.80)$ & $1.61(1.16 ; 2.23)$ & $1.43(0.98 ; 2.08)$ \\
\hline & Total & 9.8 (8.2 to 11.7$)$ & 5.7 (4.8 to 6.8 ) & $1.83(1.47 ; 2.28)$ & $1.58(1.26 ; 1.98)$ & $1.49(1.16 ; 1.91)$ \\
\hline & Total $^{\mathrm{a}}$ & $10.2(8.4$ to 12.3$)$ & 5.5 (4.5 to 6.6$)$ & N/A & $\mathrm{N} / \mathrm{A}$ & N/A \\
\hline
\end{tabular}

a) Sensitivity analysis based on self-reported antihypertensive use where the main analyses were based on ATC coding of participant medication. ${ }^{b}$ ) Age, sex, CVD history, smoking, AUDIT, BMI, Diabetes, Seen GP past year, CKD

Russia, at 10.8\% (8.1-14.1) in males and 9.2\% (7.3-11.6) in females (Table 2). The corresponding shares in Troms $\varnothing$ 7 were $6.9 \%$ (5.4-8.7) and 4.5\% (3.4-5.9). Controlling for age, gender, and CVD history, these gaps persisted, with adjusted odds ratios (AOR: KYH/Tromsø 7) of 1.65 (95\% CI 1.45-1.87) for uncontrolled hypertension and 1.58 (1.26-1.98) for aTRH (Table 2). The gaps also persisted when controlling for a wider range of co-variates (Table 2). The sensitivity analyses based on participants' response to a question about taking antihypertensives rather than the ATC-coding of self-reported medication used in the main analyses yielded similar results (Table 2).
For $\mathrm{KYH}$, the proportions with controlled hypertension (defined as blood pressures $<140 /<90 \mathrm{mmHg}$ and taking antihypertensives) out of all those with hypertension were $22.2 \%(19.6-25.1)$ for males and $43.0 \%$ (39.746.3) for females. For Tromsø 7 , the equivalent figures were similar for males at $22.4 \%$ (21.0-23.8), but lower for females, 32.6\% (30.6-34.8).

The frequency of antihypertensive use by drug class and whether hypertension was controlled or not showed that monotherapies were used in Troms $\varnothing 7$ by as many as $58 \%$ of participants, whereas in $\mathrm{KYH}$ the same proportion was only $44 \%$. While there was a declining number of participants for each additional drug class used, $\mathrm{KYH}$ had a

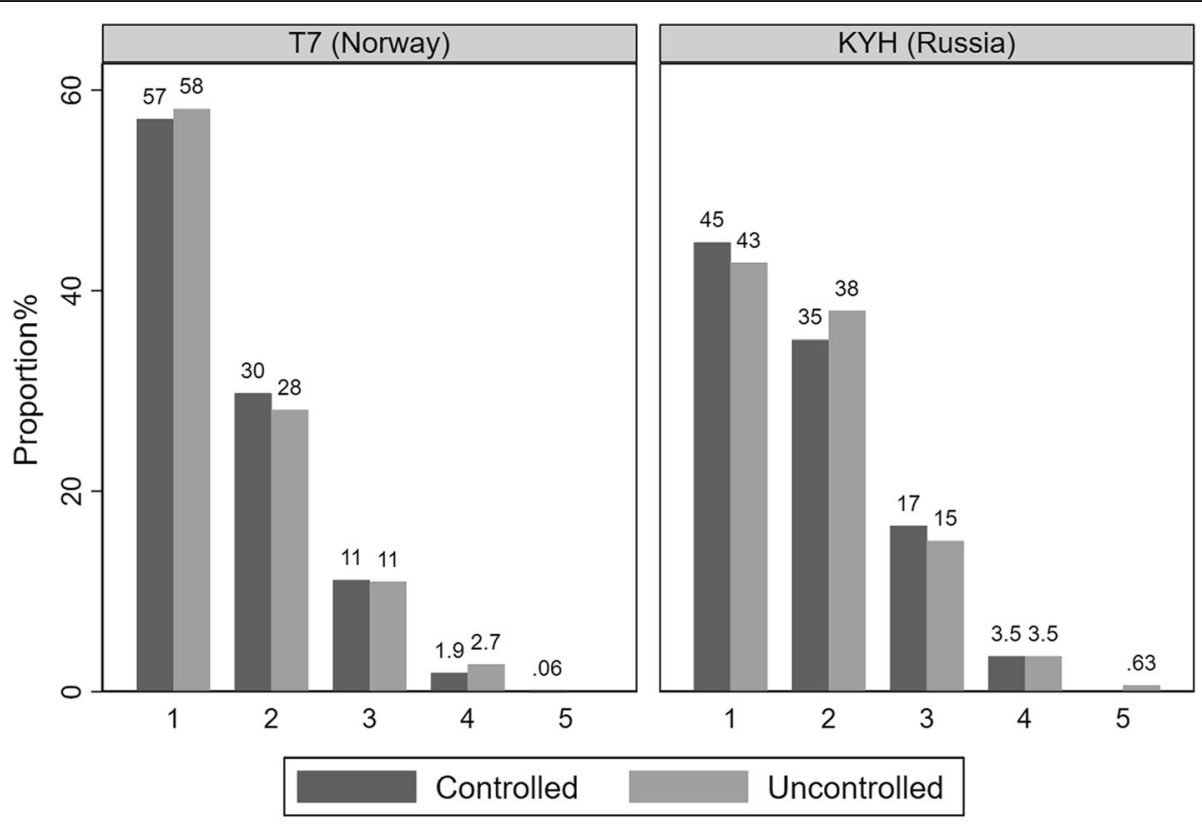

Number of Antihypertensive drug classes

Fig. 1 Number of antihypertensive drug classes used by study and whether hypertension was controlled or not 
longer right-hand tail, i.e. synonymous with greater use of multiple therapies than in Tromsø 7 (Fig. 1).

$\mathrm{ACE}$ inhibitors, $\mathrm{ARB}$, and $\mathrm{BB}$ were widely used as monotherapies in both studies; ARB (24.2\% of all with treated hypertension) was the most commonly used therapy in Troms $\varnothing 7$, while ACE (15.5\%) was the most common in $\mathrm{KYH}$ (Fig. 2). In $\mathrm{KYH}$, diuretics was used by $3.2 \%$ on its own and $22.5 \%$ were at least treated with a diuretic. In Tromsø 7, the same proportions were 5.2 and $13.1 \%$. A total of 43 participants (2.7\%) in $\mathrm{KYH}$ and $14(0.5 \%)$ in Tromsø 7 were treated with ACE and ARB concomitantly. No significant associations were found between uncontrolled hypertension status and being treated with either a monotherapy or combination therapy (Table S1).

Multivariate logistic regression models showed the following factors to be associated with uncontrolled hypertension among those treated for hypertension in both countries: older age, male gender, alcohol use disorder (AUDIT 8+), and not seeing a primary care doctor in the past year (Table 3). Additionally, the absence of CVD history was associated with uncontrolled hypertension in Tromsø 7. No significant interactions were found between gender and age group or age group and CVD history for the two outcomes in either study.

In $\mathrm{KYH}$, aTRH was associated with older age, CVD history, severe obesity (BMI 35+), diabetes, and CKD (Table 4). In Tromsø 7, aTRH was associated with older age, male gender, CVD history, any drinking, obesity (BMI 30-34) and severe obesity, and diabetes.

\section{Discussion}

The prevalence of hypertension was found to be very high in the Russian population-based study, Know Your Heart $(\mathrm{KYH})$, and this is consistent with other data from Russia $[2,3,20]$. Among those taking antihypertensives in both $\mathrm{KYH}$ and Tromsø 7, there were high proportions of individuals with uncontrolled hypertension; although higher in the Russian study (47.8\%), compared to the Norwegian study (38.2\%). The findings for KYH are consistent with a concurrent cross-sectional study of 25-64 year olds in four other regions of Russia, ESSE-RF-2 Study [3]. This study found that $50.3 \%$ of those treated for hypertension were uncontrolled.

Among all those with hypertension, blood pressure control was achieved in $22 \%$ of men (KYH and Tromsø 7), while among women it was $33 \%$ in Troms $\varnothing 7$ and $43 \%$ in KYH. The concurrent Russian ESSE-RF-2 Study found lower levels of hypertension control but similar to those in $\mathrm{KYH}$, i.e. $16.5 \%$ in males and $34.1 \%$ in females [3]. In comparison, a study with data from 123 nationally representative surveys of $40-79$ year olds in 12 high income countries [21] found that control in males ranged from $17 \%$ in Ireland to $69 \%$ in Canada. For females, from $26 \%$ in Ireland to $58 \%$ in Germany. Both $\mathrm{KYH}$ and Tromsø 7 would, in other words, be at the bottom of

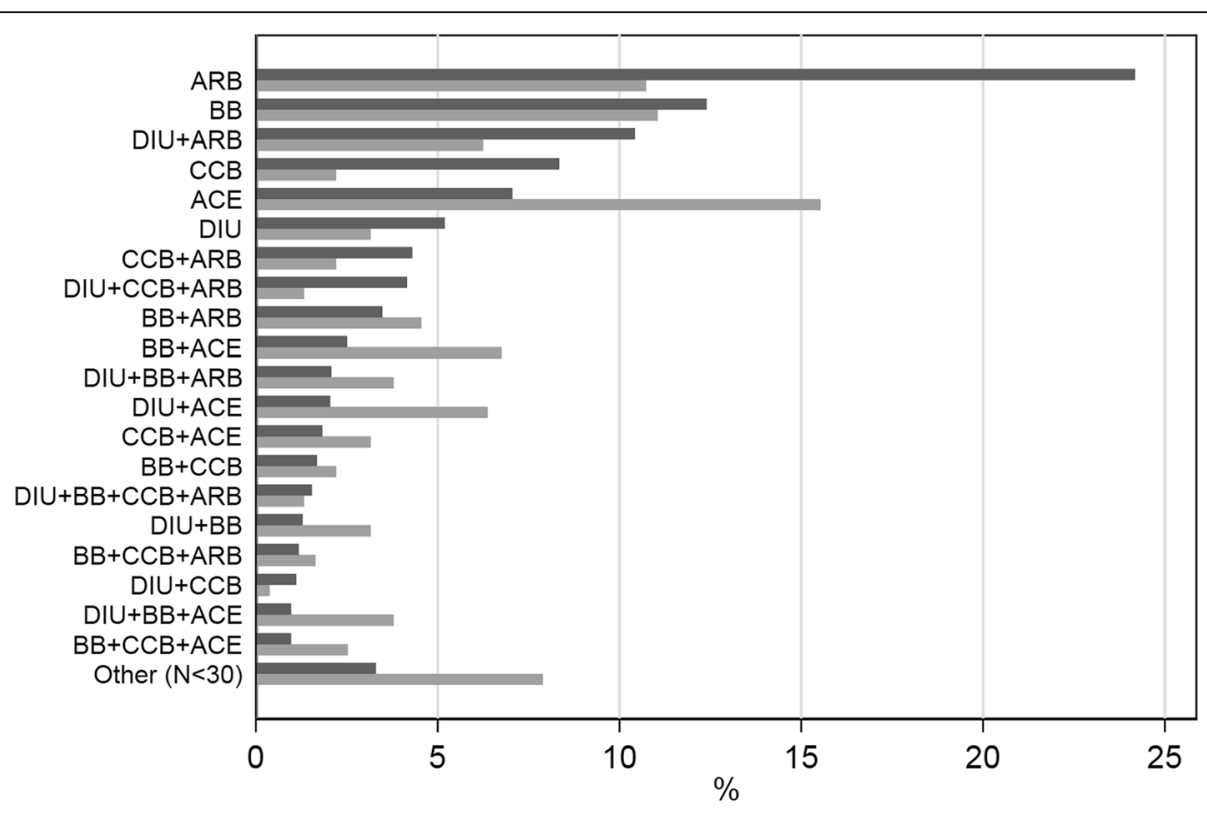

T7 (Norway)

KYH (Russia)

Fig. 2 Antihypertensive use by drug class and study. AH = Antihypertensives (ATC code: $C 02)$, DIU = Diuretics (C03), BB=Beta blockers (C07), $C C B=$ Calcium channel blockers (C08), ACE $=$ ACE inhibitors (C09A/B), ARB $=$ Angiotensin II receptor blockers (CO9C/D) 
Table 3 Logistic regression models of uncontrolled versus controlled hypertension in: Know Your Heart (KYH, Russia, $N=1584)$ relative to Tromsø 7 (T7, Norway, $N=2792)$

\begin{tabular}{|c|c|c|c|c|c|}
\hline \multirow[t]{2}{*}{ Characteristic } & \multirow[t]{2}{*}{ Level } & \multicolumn{2}{|l|}{ KYH Russia } & \multicolumn{2}{|l|}{ T7 Norway } \\
\hline & & AOR gender/age & AOR gender/age/CVD & AOR gender/age & AOR gender/age/CVD \\
\hline \multirow[t]{3}{*}{ Age group } & $40-49 y r$ & Ref & & Ref & \\
\hline & $50-59 y r$ & $1.25(0.90 ; 1.74)$ & & $1.13(0.88 ; 1.44)$ & \\
\hline & $60-69 y r$ & $1.45(1.07 ; 1.98)$ & & $\mathbf{1 . 5 3}(1.22 ; 1.91)$ & \\
\hline \multirow[t]{2}{*}{ Gender } & Female & Ref & & Ref & \\
\hline & Male & $1.82(1.48 ; 2.24)$ & & $1.25(1.08 ; 1.46)$ & \\
\hline \multirow[t]{2}{*}{ CVD history } & No & Ref & & Ref & \\
\hline & Yes & $0.83(0.67 ; 1.02)$ & & $\mathbf{0 . 6 5}(0.54 ; 0.78)$ & \\
\hline \multirow[t]{4}{*}{ Education } & Elementary & Ref & Ref & Ref & Ref \\
\hline & Lower intermediate & $0.97(0.67 ; 1.42)$ & $0.97(0.67 ; 1.43)$ & $0.92(0.75 ; 1.12)$ & $0.91(0.74 ; 1.11)$ \\
\hline & Higher intermediate & $0.79(0.58 ; 1.07)$ & $0.79(0.58 ; 1.07)$ & $0.96(0.75 ; 1.21)$ & $0.96(0.76 ; 1.21)$ \\
\hline & Graduate & $0.79(0.58 ; 1.08)$ & $0.78(0.57 ; 1.07)$ & $0.81(0.65 ; 1.02)$ & $0.81(0.64 ; 1.00)$ \\
\hline \multirow[t]{3}{*}{ Financial constraints } & Constrained & Ref & Ref & Ref & Ref \\
\hline & Intermediary & $0.97(0.75 ; 1.24)$ & $0.95(0.74 ; 1.23)$ & N/A & N/A \\
\hline & Rel. unconstrained & $0.96(0.72 ; 1.29)$ & $0.94(0.70 ; 1.26)$ & N/A & N/A \\
\hline \multirow[t]{2}{*}{ Single } & No & Ref & Ref & Ref & Ref \\
\hline & Yes & $0.95(0.76 ; 1.19)$ & $0.96(0.77 ; 1.21)$ & $1.06(0.88 ; 1.27)$ & $1.07(0.89 ; 1.43)$ \\
\hline \multirow[t]{2}{*}{ Smoking } & No & Ref & Ref & Ref & Ref \\
\hline & Yes & $0.92(0.71 ; 1.19)$ & $0.92(0.71 ; 1.20)$ & $0.81(0.64 ; 1.02)$ & $0.83(0.65 ; 1.05)$ \\
\hline \multirow[t]{3}{*}{ Alcohol use disorder } & Non-drinker past year & Ref & Ref & Ref & Ref \\
\hline & Low (AUDIT<8) & $1.13(0.85 ; 1.79)$ & $1.11(0.84 ; 1.47)$ & $1.16(0.97 ; 1.39)$ & $1.16(0.97 ; 1.39)$ \\
\hline & High (AUDIT 8+) & $1.71(1.15 ; 2.54)$ & $1.69(1.13 ; 2.51)$ & $1.35(1.04 ; 1.74)$ & $1.37(1.06 ; 1.78)$ \\
\hline \multirow[t]{4}{*}{ Body Mass Index } & Under/Normal $(<25)$ & Ref & Ref & Ref & Ref \\
\hline & Overweight (25-29) & $1.32(0.97 ; 1.81)$ & $1.32(0.96 ; 1.80)$ & $1.03(0.83 ; 1.29)$ & $1.03(0.83 ; 1.28)$ \\
\hline & Obese (30-34) & $1.32(0.96 ; 1.82)$ & $1.32(0.96 ; 1.82)$ & $1.16(0.92 ; 1.47)$ & $1.16(0.92 ; 1.47)$ \\
\hline & Very obese (35+) & $1.19(0.83 ; 1.69)$ & $1.19(0.84 ; 1.71)$ & $1.28(0.96 ; 1.70)$ & $1.26(0.95 ; 1.68)$ \\
\hline \multirow[t]{2}{*}{ Diabetic } & No & Ref & Ref & Ref & Ref \\
\hline & Yes & $0.93(0.74 ; 1.18)$ & $0.95(0.75 ; 1.19)$ & $1.03(0.83 ; 1.27)$ & $1.03(0.83 ; 1.27)$ \\
\hline \multirow[t]{2}{*}{ Seen GP past year } & No & Ref & Ref & Ref & Ref \\
\hline & Yes & $\mathbf{0 . 5 8}(0.44 ; 0.75)$ & $\mathbf{0 . 5 9}(0.45 ; 0.76)$ & $\mathbf{0 . 6 7}(0.50 ; 0.90)$ & $\mathbf{0 . 6 7}(0.50 ; 0.91)$ \\
\hline \multirow[t]{2}{*}{ CKD } & No & Ref & Ref & Ref & Ref \\
\hline & Yes & $1.25(0.76 ; 2.08)$ & $1.27(0.77 ; 2.08)$ & $1.09(0.73 ; 1.65)$ & $1.11(0.74 ; 1.68)$ \\
\hline
\end{tabular}

the range, except that females in $\mathrm{KYH}$ would be in the middle of this range.

Monotherapies were widely used in both countries, $58 \%$ in Tromsø 7 and $44 \%$ in $\mathrm{KYH}$. This is contrary to the current European treatment guidelines, which recommend a diuretic to be combined with a drug acting on the renin system (ACE or ARB) or that at least two different drug classes are to be combined [6]. For comparison, the prevalence for monotherapy use in the US (2009-2014) was only $37 \%$ [12].

Those with uncontrolled hypertension were less likely to have visited a primary care doctor in the past year than those with controlled hypertension. Poor adherence to antihypertensive therapies may also play a role in the levels of uncontrolled hypertension observed in both studies. Many of the factors found to be associated with uncontrolled hypertension overlap with known factors for poor drug adherence $[19,22]$ and health check non-attendance [23], i.e. male gender, no primary care visit in the past year, problem drinking, and absence of CVD history. Similarly, poor adherence to lifestyle changes in terms of e.g. weight loss, exercise, dietary salt reduction, etc. could compound the effectiveness of hypertension control for individuals. A recent review of Russian hypertension 
Table 4 Logistic regression models of aTRH versus non-aTRH in: Know Your Heart (KYH, Russia, N=1584) relative to Troms $\varnothing 7$ (T7, Norway, $N=2792$ )

\begin{tabular}{|c|c|c|c|c|c|}
\hline \multirow[t]{2}{*}{ Characteristic } & \multirow[t]{2}{*}{ Level } & \multicolumn{2}{|l|}{ KYH Russia } & \multicolumn{2}{|l|}{ T7 Norway } \\
\hline & & AOR gender/age & AOR gender/age/CVD & AOR gender/age & AOR gender/age/CVD \\
\hline \multirow[t]{3}{*}{ Age group } & $40-49 y r$ & Ref & & Ref & \\
\hline & $50-59 y r$ & $1.00(0.56 ; 1.81)$ & & $1.52(0.84 ; 2.77)$ & \\
\hline & $60-69 y r$ & $1.77(1.04 ; 3.02)$ & & $2.47(1.43 ; 4.27)$ & \\
\hline \multirow[t]{2}{*}{ Gender } & Female & Ref & & Ref & \\
\hline & Male & $1.12(0.81 ; 1.53)$ & & $1.41(1.04 ; 1.91)$ & \\
\hline \multirow[t]{2}{*}{ CVD history } & No & Ref & & Ref & \\
\hline & Yes & $2.42(1.74 ; 3.37)$ & & $1.78(1.29 ; 3.89)$ & \\
\hline \multirow[t]{4}{*}{ Education } & Elementary & Ref & Ref & Ref & Ref \\
\hline & Lower intermediate & $1.06(0.59 ; 1.89)$ & $1.03(0.57 ; 1.85)$ & $0.81(0.54 ; 1.21)$ & $0.82(0.55 ; 1.22)$ \\
\hline & Higher intermediate & $1.09(0.69 ; 1.74)$ & $1.08(0.68 ; 1.72)$ & $0.89(0.57 ; 1.41)$ & $0.89(0.57 ; 1.41)$ \\
\hline & Graduate & $0.97(0.59 ; 1.58)$ & $1.03(0.63 ; 1.69)$ & $1.17(0.77 ; 1.76)$ & $1.18(0.78 ; 1.78)$ \\
\hline \multirow[t]{3}{*}{ Financial constraints } & Constrained & Ref & Ref & Ref & Ref \\
\hline & Intermediary & $0.99(0.67 ; 1.47)$ & $1.06(0.71 ; 1.58)$ & N/A & N/A \\
\hline & Rel. unconstrained & $1.11(0.71 ; 1.75)$ & $1.29(0.82 ; 2.05)$ & N/A & N/A \\
\hline \multirow[t]{2}{*}{ Single } & No & Ref & Ref & Ref & Ref \\
\hline & Yes & $0.80(0.56 ; 1.15)$ & $0.77(0.53 ; 1.11)$ & $1.17(0.82 ; 1.67)$ & $1.15(0.80 ; 1.65)$ \\
\hline \multirow[t]{2}{*}{ Smoking } & No & Ref & Ref & Ref & Ref \\
\hline & Yes & $0.69(0.43 ; 1.06)$ & $0.68(0.43 ; 1.06)$ & $0.77(0.46 ; 1.27)$ & $0.73(0.44 ; 1.22)$ \\
\hline \multirow[t]{3}{*}{ Alcohol use disorder } & Non-drinker past year & Ref & Ref & Ref & Ref \\
\hline & Low (AUDIT< <) & $0.93(0.59 ; 1.46)$ & $1.02(0.64 ; 1.60)$ & $1.44(1.01 ; 2.05)$ & $1.45(1.02 ; 2.07)$ \\
\hline & High (AUDIT 8+) & $1.00(0.55 ; 1.81)$ & $1.10(0.60 ; 2.02)$ & $1.65(1.03 ; 2.65)$ & $1.63(1.01 ; 2.63)$ \\
\hline \multirow[t]{4}{*}{ Body Mass Index } & Under/Normal $(<25)$ & Ref & Ref & Ref & Ref \\
\hline & Overweight (25-29) & $1.28(0.71 ; 2.30)$ & $1.32(0.73 ; 2.37)$ & $1.28(0.76 ; 2.14)$ & $1.28(0.76 ; 2.16)$ \\
\hline & Obese (30-34) & $1.73(0.97 ; 3.10)$ & $1.75(0.97 ; 3.15)$ & $1.94(1.15 ; 3.29)$ & $1.95(1.15 ; 3.31)$ \\
\hline & Very obese (35+) & $3.15(1.73 ; 5.73)$ & $3.10(1.70 ; 5.68)$ & $\mathbf{3 . 8 4}(2.19 ; 6.72)$ & $3.97(2.27 ; 6.97)$ \\
\hline \multirow[t]{2}{*}{ Diabetic } & No & Ref & Ref & Ref & Ref \\
\hline & Yes & $2.40(1.74 ; 3.32)$ & $2.31(1.67 ; 3.26)$ & $2.31(1.64 ; 3.24)$ & $2.32(1.65 ; 3.27)$ \\
\hline \multirow[t]{2}{*}{ Seen GP past year } & No & Ref & Ref & Ref & Ref \\
\hline & Yes & $1.26(0.82 ; 1.92)$ & $1.14(0.74 ; 1.75)$ & $1.43(0.74 ; 2.75)$ & $1.42(0.73 ; 2.74)$ \\
\hline \multirow[t]{2}{*}{ CKD } & No & Ref & Ref & Ref & Ref \\
\hline & Yes & $2.29(1.27 ; 4.13)$ & $\mathbf{2 . 2 0}(1.21 ; 4.00)$ & $1.56(0.81 ; 3.00)$ & $1.52(0.79 ; 2.93)$ \\
\hline
\end{tabular}

research found evidence that lower education and single status were associated with lower adherence but differences were not significant in this study [19], perhaps because of insufficient power to detect such a difference.

The prevalence of aTRH was $9.8 \%$ in $\mathrm{KYH}$ and $5.7 \%$ in Tromsø 7. For comparison, the prevalences of aTRH were $14.5 \%$ in a US population-based study (2005-2008) [11] and $6.4 \%$ in a UK primary care database study [24]. The UK and US studies included those aged above 70 years and may thus be inflated relative to the present study of 40-69 year olds.
The factors associated with aTRH were indicative of patient level factors, i.e. CVD history, obesity, and diabetes. These associations are well-known in the literature, as hypertension is harder to control in patients with obesity and some co-morbidities [6,11]. Patients with CKD are also known to be at risk of circulatory system conditions including aTRH [11]. The odds ratios for CKD was found statistically significant in $\mathrm{KYH}$, but not in Tromsø 7 .

There were some differences in the factors associated with aTRH between the two countries. The associations with CVD history was stronger in Russia than in Norway. This suggests that the Russian participants with aTRH 
had higher levels of comorbidity. There was only an association of aTRH with alcohol use disorder in Norway. One possible explanation for this difference could be a higher proportion of 'sick quitters' in the Russian study population, i.e. individuals who stop a health harming behaviour upon diagnosis with a related health condition.

Interventions to improve hypertension control confront different challenges and opportunities in these two countries. Russia has a general health check programme [5], while Norway does not. In Norway antihypertensive medicines are only available on prescription, whereas in Russia it is possible to obtain any marketable antihypertensive from a pharmacy over-the-counter (OTC). A drug may thus be taken either because it was prescribed or the patient chose, perhaps on the recommendation of a pharmacist, to purchase it [7]. Moreover, patients with hypertension in Norway are reimbursed most of the prescriptions costs [25], while only certain groups including war veterans and recipients of the minimum state pension are reimbursed in Russia [7].

Pharmacists play a particularly important role in the Russian health system, something only a few studies have to date looked at, such as the quality of advice given to patients and opportunities for follow-up.

Antihypertensive combinations that included both ACE inhibitors and ARB were used in both study populations contrary to European treatment guidelines [6]. These two agents both act through the renin-angiotensin system and as a combination not as effective as if patients are treated with drugs with complementary mechanisms. Patients using this combination are also more likely to experience adverse renal events including kidney failure [6]. In absolute terms, this contraindicated co-prescription was relatively rare; $2.7 \%$ in the Russian study and $0.5 \%$ in the Norwegian one. Electronic prescribing, which includes alerts to potential problems, is used in primary care and pharmacies in Norway, which may explain why this combination was rarer in Norway than Russia. Greater use of multi-drug combinations could potentially reduce prescription errors and improve adherence by reducing the "pill burden" for patients [26], but typically reduces treatment options in terms of dose, formulation, and more individualised prescribing. A recent review of Russian antihypertensive adherence studies however concluded that patient education, telephone reminders, home blood pressure monitoring, and fixed drug combinations were the most important factors for improving adherence [19].

A range of lifestyle factors are associated with hypertension and are assessed as part of standard medical advice, e.g. initiating weight loss, increasing exercise, and reducing dietary salt intake $[27,28]$. Adherence to lifestyle change recommendations is thus another potential avenue for future research [29].
Analysis of different therapies by aTRH status was limited in this study by the relatively small sample size and should be replicated in a large database study to properly assess the treatment regimens for this patient group.

\section{Limitations}

Sampling bias introduced by non-response in KYH was assessed by comparing the realised sample against data from the Russian Census 2010 on age, gender, and higher education attainment [8]. Overall, the realised sample for the health check was close to equity, ratio of 0.99 (95\% CI 0.93-1.06) for Arkhangelsk and 1.26 (1.17-1.34) for Novosibirsk.

Determination of uncontrolled hypertension and treatment resistance in a survey differs from that in clinical practice, where the treatment regimen, any side effects, and adherence would be reviewed together, perhaps with advice on other actions such as home blood pressure monitoring. This is a limitation that should be borne in mind when interpreting the findings from this and similar studies.

Blood pressure can be spuriously elevated in apprehensive individuals, the so-called white coat effect [30]. This is a type of measurement error is an inevitable limitation of studies such as this where all measurements are done in a clinical setting in a single sitting. To reduce bias from this source, only the mean of the last two of three measurements was analysed.

Antihypertensive use was based in part on self-reported medication data and, as such, potentially prone to recall and other reporting biases. There is however evidence in the literature that self-reports of CVD medication are accurate $[31,32]$.

\section{Conclusion}

There were high levels of uncontrolled hypertension in both countries, although more so in the Russian than the Norwegian study population. Antihypertensive monotherapies were commonly used in both countries counter to European treatment guidelines, especially in Norway. Our findings suggest considerable scope for promoting the use of combination therapies for those uncontrolled on a single drug. The relatively high proportions of patients not controlled despite being on multiple antihypertensive drugs, furthermore, points to the need to invite patients for individual review of their treatment and any barriers they face adhering to treatment. Further studies should thus include non-adherence in those with uncontrolled hypertension as well as a more in-depth study of patients with aTRH.

\section{Supplementary information}

Supplementary information accompanies this paper at https://doi.org/10. 1186/s12872-020-01407-2. 
Additional file 1: Figure S1. Sample selection flow diagram. Hypertension was defined as self-reported antihypertensive use or high blood pressure $(140+/ 90+$ mmHg). Table S1. Age- and gender-adjusted odds ratios (AOR) of uncontrolled versus controlled hypertension and the association with antihypertensive drug class combinations by study: Know Your Heart (KYH, Russia) and Tromsø 7 (T7, Norway).

\section{Abbreviations}

ACE: ACE inhibitors ATC code C09A/B; AH: Antihypertensives ATC code C02; ARB: Angiotensin II receptor blockers ATC code C09C/D; ATC: International WHO anatomical therapeutic chemical classification system; aTRH: Apparent treatment resistant hypertension; AUDIT: WHO alcohol use disorders identification test; BB: Beta blockers ATC code C07; CCB: Calcium channel blockers ATC code C08; CKD: Chronic kidney disease; CVD: Cardiovascular disease; DIU: Diuretics ATC code C03; eGFR: Glomerular filtration rate; $\mathrm{KYH}$ : Know your heart Study; OTC: Over the counter

\section{Acknowledgements}

\section{Not applicable.}

\section{Authors' contributions}

All authors contributed to conception and design, critically revised the manuscript, gave final approval, and agreed to be accountable for all aspects of work ensuring integrity and accuracy (JP, SM, AR, AKO, AKU, AEE,MM, SC, $\mathrm{LAH}, \mathrm{HS}, \mathrm{DAL}$ ). JP contributed to data acquisition, analysis, and the first draft of the manuscript.

\section{Funding}

Know Your Heat $(\mathrm{KYH})$ is a component of International Project on Cardiovascular Disease in Russia (IPCDR) and funded by Wellcome Trust Strategic Award [100217], UiT The Arctic University of. Norway (UiT), Norwegian Institute of Public Health, and Norwegian Ministry of Health and Social Affairs. Funding for the Tromsø Study was obtained from UiT The Arctic University of Norway, Northern Norway Regional Health Authority, Ministry of Health and Care Services, Norwegian Research Council, and various public and charity research funds in Norway. The funding bodies have no role in the design of the study, data collection, analysis, interpretation of data, or in writing the manuscript.

\section{Availability of data and materials}

The data that support the findings of this study are available from Know Your Heart and The Tromsø Study, but restrictions apply to the availability of these data, which were used under license for the current study, and so are not publicly available. Data from the Know Your Heart Study are however available from the authors upon reasonable request and with permission of Know Your Heart [33]. For The Tromsø Study, data are available subject to scientific and ethical approval of a study protocol [9].

\section{Ethics approval and consent to participate}

Ethical approval for $\mathrm{KYH}$ was received from the ethics committees of the London School of Hygiene \& Tropical Medicine (approval number 8808), Novosibirsk State Medical University (approval number 75; 21 May 2015), the Institute of Preventative Medicine (approval received 26 December 2014), Novosibirsk and the Northern State Medical University, Arkhangelsk (approval number 01/01-15; 27 January 2015). Tromsø 7 obtained ethical approval from Regional Committee of Medical and Health Research Ethics (REC North) (approval number 2014/940) and Norwegian Data Protection Authority, and was conducted in accordance with the 1964 Helsinki Declaration and its later amendments. Participants gave written informed consent.

\section{Consent for publication}

Not applicable.

\section{Competing interests}

HS have received lecture fees from pharmaceutical companies with market authorisation for antihypertensives (Novartis, MSD, Astra Zeneca, Sanofi Aventis). Other authors have no competing interests to declare (SM, AR, AKo, AKu, AEE, MM, SC, LAH, DL, JP).

\section{Author details}

${ }^{1}$ London School of Hygiene \& Tropical Medicine, WC1E 7HT, London, UK. ${ }^{2}$ Research Institute of Internal and Preventive Medicine, Branch of Institute of Cytology and Genetics, Siberian Branch of the Russian Academy of Sciences, Novosibirsk 630090, Russia. ${ }^{3}$ Novosibirsk State Medical University, Russian Ministry of Health, Novosibirsk 630091, Russia. ${ }^{4}$ National Research Center for Preventive Medicine, Ministry of Healthcare, Moscow, Russia. ${ }^{5}$ Northern State Medical University, Arkhangelsk 163000, Russia. ${ }^{6}$ Department of Community Medicine, UiT The Arctic University of Norway, 9037 Tromsø, Norway. ${ }^{7}$ University of Oslo, Institute for clinical medicine, 1171 Blindern, 0318 Oslo, Norway. ${ }^{8}$ Department of Cardiology, Akershus University Hospital, 1478 Nordbyhagen, Oslo, Norway. ${ }^{9}$ International Laboratory for Population and Health, National Research University, Higher School of Economics, Moscow, Russia.

Received: 13 November 2019 Accepted: 28 February 2020

Published online: 13 March 2020

\section{References}

1. Timmis A, Townsend N, Gale C, Grobbee R, Maniadakis N, Flather M, et al. European Society of Cardiology: cardiovascular disease statistics 2017. Eur Heart J. 2018:39:508-79.

2. Shalnova SA, Deev AD, Balanova YA, Kapustina AV, Imaeva AE, Muromtseva $\mathrm{GA}$, et al. Twenty years trends of obesity and arterial hypertension and their association in Russia. Cardiovasc Ther Prev Russ Fed. 2017;16:4-10..

3. Balanova YA, Shalnova S, Imaeva AE, Kapustina AV, Muromtseva GA, Evstifeeva SV, et al. Prevalence, awareness, treatment and control of hypertension in Russian Federation (data of observational ESSERF-2 Study). Ration Pharmacother Cardiol. 2019;15:450-65.

4. Kontsevaya A, Shalnova S, Deev A, Breda J, Jewell J, Rakovac I, et al. Overweight and obesity in the Russian population: prevalence in adults and association with socioeconomic parameters and cardiovascular risk factors. Obes Facts. 2019;12:103-14.

5. Sheiman I, Shishkin S, Shevsky V. The evolving Semashko model of primary health care: the case of the Russian Federation. Risk Manag Healthc Policy. 2018;11:209-20.

6. Williams B, Mancia G, Spiering W, Agabiti Rosei E, Azizi M, Burnier M, et al. 2018 practice guidelines for the management of arterial hypertension of the European Society of Cardiology and the European Society of Hypertension. Blood Press. 2018;27:314-40.

7. Imaeva AE, Balanova YA, Kontsevaya AV, Kapustina AV, Duplyakov DV, Malysheva $\mathrm{OH}$, et al. Availability and Affordability of Medicines for the Treatment of Cardiovascular Diseases in Pharmacies in Six Regions of the Russian Federation. Ration Pharmacother Cardiol. 2018;14(6):804-15. https:// www.rpcardio.com/jour/article/view/1787. Accessed 23 May 2019.

8. Cook S, Malyutina S, Kudryavtsev AV, Averina M, Bobrova N, Boytsov S, et al. Know your heart: rationale, design and conduct of a cross-sectional study of cardiovascular structure, function and risk factors in 4500 men and women aged 35-69 years from two Russian cities, 2015-18. Wellcome Open Res. 2018:3:67.

9. Tromsø Study. Tromsøundersøkelsen: Tromsø 7. 2019. https://uit.no/ forskning/forskningsgrupper/sub?p_document_id=367276\&sub_id=503778. Accessed 18 Sep 2019.

10. WHOCC. WHOCC - ATC/DDD Index. 2019. https://www.whocc.no/atc_ddd index/. Accessed 18 Sep 2019.

11. Carey RM, Calhoun DA, Bakris GL. Resistant hypertension: detection, evaluation, and management: a scientific statement from the American Heart Association | hypertension. 2018. https://www.ahajournals.org/doi/full/ https://doi.org/10.1161/HYP.0000000000000084. Accessed 24 May 2019.

12. Carey RM, Sakhuja S, Calhoun DA, Whelton PK, Muntner P. Prevalence of Apparent Treatment-Resistant Hypertension in the United States. Hypertens Dallas Tex. 2019;73:424-31.

13. Eurostat. Revision of the European Standard Population - Report of Eurostat's task force 2013 edition. 2013. https://ec.europa.eu/eurostat/web/ products-manuals-and-guidelines/-/KS-RA-13-028. Accessed 21 May 2019.

14. Inker LA, Schmid CH, Tighiouart H, Eckfeldt JH, Feldman HI, Greene T, et al. Estimating glomerular filtration rate from serum Creatinine and Cystatin C. N Engl J Med. 2012;367:20-9.

15. Babor T, Higgins-Biddle J, Saunders J. The alcohol use disorders identification test: guidelines for use in primary care: World Health Organization (WHO); 2001. 
16. Moody A, Cowley G, Fat LN, Mindell JS. Social inequalities in prevalence of diagnosed and undiagnosed diabetes and impaired glucose regulation in participants in the health surveys for England series. BMJ Open. 2016;6: e010155.

17. Conroy RM, Pyörälä K, Fitzgerald AP, Sans S, Menotti A, De Backer G, et al. Estimation of ten-year risk of fatal cardiovascular disease in Europe: the SCORE project. Eur Heart J. 2003;24:987-1003.

18. StataCorp. Stata Statistical Software: Release 15. 2017.

19. Bochkareva EV, Butina EK, Kim IV, Kontsevaya AV, Drapkina OM, Leon DA, et al. Adherence to antihypertensive medication in Russia: a scoping review of studies on levels, determinants and intervention strategies published between 2000 and 2017. Arch Public Health. 2019;77.

20. Pajak A, Szafraniec K, Kubinova R, Malyutina S, Peasey A, Pikhart H, et al. Binge drinking and blood pressure: cross-sectional results of the HAPIEE study. PLoS One. 2013;8:e65856.

21. Zhou B, Danaei G, Stevens GA, Bixby H, Taddei C, Carrillo-Larco RM, et al. Long-term and recent trends in hypertension awareness, treatment, and control in 12 high-income countries: an analysis of 123 nationally representative surveys. Lancet. 2019. https://doi.org/10.1016/S01406736(19)31145-6.

22. Sabaté E. Adherence to long-term therapies: evidence for action. Geneva: World Health Organization; 2003.

23. Dryden R, Williams B, McCowan C, Themessl-Huber M. What do we know about who does and does not attend general health checks?. Findings from a narrative scoping review. BMC Public Health. 2012;12:723.

24. Sinnott S-J, Smeeth L, Williamson E, Douglas IJ. Trends for prevalence and incidence of resistant hypertension: population based cohort study in the UK 1995-2015. BMJ. 2017;358:j3984.

25. Statens Legemiddelverk. The Norwegian medicines agency Legemiddelverket: Statens legemiddelverk; 2019. https://legemiddelverket. no/English. Accessed 19 Sep 2019.

26. Hameed MA, Dasgupta I. Medication adherence and treatment-resistant hypertension: a review. Drugs Context. 2019;8:212560.

27. Vartiainen E. The North Karelia Project: Cardiovascular disease prevention in Finland. Glob Cardiol Sci Pract. 2018. https://doi.org/10.21542/gcsp.2018.13.

28. Yang Q, Cogswell ME, Flanders WD, Hong Y, Zhang Z, Loustalot F, et al. Trends in cardiovascular health metrics and associations with all-cause and CVD mortality among US adults. JAMA. 2012;307:1273-83.

29. Spring B, Moller AC, Coons MJ. Multiple health behaviours: overview and implications. J Public Health Oxf Engl. 2012;34(Suppl 1):i3-10.

30. Paini A, Bertacchini F, Stassaldi D, Aggiusti C, Maruelli G, Arnoldi C, et al. Unattended versus attended blood pressure measurement: mean values and determinants of the difference. Int J Cardiol. 2019;274:305-10.

31. Sediq R, van der Schans J, Dotinga A, Wiffert B, Bos JH, et al. Concordance assessment of self-reported medication use in the Netherlands threegeneration Lifelines Cohort study with the pharmacy database iaDB.nl: The PharmLines initiative. Clin Epidemiol. 2018;10:981-9.

32. Drieling RL, LaCroix AZ, Beresford SA, Boudreau DM, Kooperberg C, Heckbert SR. Validity of self-reported medication use compared with pharmacy Records in a Cohort of older women: findings from the Women's Health Initiative. Am J Epidemiol. 2016 Aug 1;184(3):233-8. https://doi.org/ 10.1093/aje/kwv446.

33. Know Your Heart. Know Your Heart: Узнай своё сердце: Users. 2019. https://metadata.knowyourheart.science/. Accessed 12 Nov 2019.

\section{Publisher's Note}

Springer Nature remains neutral with regard to jurisdictional claims in published maps and institutional affiliations.

Ready to submit your research? Choose BMC and benefit from:
- fast, convenient online submission
- thorough peer review by experienced researchers in your field
- rapid publication on acceptance
- support for research data, including large and complex data types
- gold Open Access which fosters wider collaboration and increased citations
- maximum visibility for your research: over 100M website views per year
At BMC, research is always in progress.
Learn more biomedcentral.com/submissions

